\title{
XLV. Experimental researches in electricity.-Eighth series
}

\section{Michael Faraday D.C.L. F.R.S.}

To cite this article: Michael Faraday D.C.L. F.R.S. (1835) XLV. Experimental researches in electricity.-Eighth series, Philosophical Magazine Series 3, 6:34, 272-279, DOI: 10.1080/14786443508648594

To link to this article: http://dx.doi.org/10.1080/14786443508648594

册 Published online: 01 Jun 2009.

Submit your article to this journal $[\pi$

ЏII Article views: 6

Q View related articles $\asymp$ 
272 Dr. Faraday's Experimental Researches in Electricity.

initial arbitrary discontinuity of the motion. The general proposition proved above renders it unnecessary to have recourse to this method.

To complete this subject it will be proper to determine what takes place when the propagated motion reaches the fixed extremity of the chord. Suppose two propagations to take place in opposite directions, being exactly like in every respect excepting that the ordinates in one are of a contrary sign to those in the other. The point where the opposite propagations meet, being affected by equal and opposite motions, will remain constantly at rest. Nothing will be altered if this be supposed a fixed point, and the portion of the chord on one side be removed. We have then the case of a propagation reflected from a fixed extremity of the chord, and the manner in which it will take place is easily inferred from these considerations. This proposition being proved, the principles advocated in this paper, suffice for solving all the questions commonly proposed respecting the vibrations of elastic chords.

Papworth St. Everard, Jan. 19, 1835.

XLV. Experimental Researches in Electricity.-Eighth Series. By Michaex. Faraday, D.C.L.F.R.S. Fullerian Prof. Chem. Royal Institution, Corr. Memb. Royal and Imp. Acadd. of Sciences, Paris, Petersburgh, Florence, Copenhagen, Berlin, \&c.\&c.

[Continued from p. 182.]

\section{I ii. On the Intensity necessary for Electrolyzation.}

966. I $\mathrm{T}$ became requisite, for the comprehension of many of the conditions attending voltaic action, to determine positively, if possible, whether electrolytes could resist the action of an electric current if beneath a certain intensity? whether the intensity at which the current ceased to act would be the same for all bodies? and also whether the electrolytes thus resisting decomposition would conduct the electric current as a metal does, after they ceased to conduct as electrolytes, or would act as perfect insulators?

967. It was evident from the experiments described (904. 906.) that different bodies were decomposed with very different facilities, and apparently that they required for their decomposition currents of different intensities, resisting some, but giving way to others. But it was needful, by very careful and express experiments, to determine whether a current 
could really pass through, and yet not decompose an electrolyte (910.).

968. An arrangement (fig. 12.) was made, in which two glass vessels contained the same dilute sulphuric acid, sp. gr. $1 \cdot 25$. The plate $z$ was amalgamated zinc, in connexion, by a platina wire $a$, with the platina plate $e ; b$ was a platina wire connecting the two platina plates $\mathrm{P} \mathrm{P}^{\prime} ; c$ was a platina wire connected with the platina plate $\mathrm{P}^{\prime \prime}$. On the plate $e$ was placed a piece of paper moistened in solution of iodide of potassium: the wire $c$ was so curved that its end could be made to rest at pleasure on this paper, and show, by the evolution of iodine there, whether a current was passing; or, being placed in the dotted position, it formed a direct communication with the platina plate $e$, and the electricity could pass without causing decomposition. The object was to produce a current by the action of the acid on the amalgamated zinc in the first vessel; to pass it through the acid in the second vessel by platina electrodes, that its power of decomposing water might, if existing, be observed; and to verify the existence of the current at pleasure, by decomposition at $e$, without involving the continual obstruction to the current which would arise from making the decomposition there con. stant. The experiment, being arranged, was examined, the existence of a current shown by the decomposition at $e$, and then left with the end of the wire $c$ resting on the plate $e$, so as to form a constant metallic communication there.

969. After several hours, the end of the wire $c$ was replaced on the test paper at $e$ : decomposition occurred, and the proof of a passing current was therefore complete. The current was very feeble compared to what it had been at the beginning of the experiment, because of a peculiar state acquired by the metal surfaces in the second vessel, which caused them to oppose the passing current by a force which they possess under these circumstances (1040.). Still it was proved, by the decomposition, that this state of the plates in the second vessel was not able entirely to stop the current determined in the first, and that was all that was needful to be ascertained in the present inquiry.

970. This apparatus was examined from time to time, and an electric current always found circulating through it, until twelve days had elapsed, during which the water in the second vessel had been constantly subject to its action. Notwithstanding this lengthened period, not the slightest appearance of a bubble upon either of the plates in that vessel nccurrerl. From the results of the experinent, I conclude that a current had passed, but of so low an intensity as to fall beThird Series. Vol. 6. No. 34. April 1835. $2 \mathrm{~N}$ 


\section{Dr. Faraday's Expcrimental Researches in Electricity.}

neath that degree at which the elements of water, unaided by any secondary force resulting from the capability of combination with the matter of the electrodes, or of the liquid surrounding them, separated from each other.

971. It may be supposed, that the oxygen and hydrogen had been evolved in such small quantities as to have entirely dissolved in the water, and finally to have escaped at the surface, or to have reunited into water. That the hydrogen can be so dissolved was shown in the first vessel; for after several days minute bubbles of gas gradually appeared upon a glass rod, inserted to retain the zinc and platina apart, and also upon the platina plate itself, and these were hydrogen. They resulted in this way. Notwithstanding the amalgamation of the zinc, the acid exerted a little direct action upon it, so that a small stream of hydrogen bubbles was continually rising from its surface; a little of this hydrogen gradually dissolved in the dilute acid, and was in part set free against the surfaces of the rod and the plate, according to the wellknown action of such solid bodies in solutions of gases (623. $\& c$.$) .$

972. But if the gases had been evolved in the second vessel by the decomposition of water, and had tended to dissolve, still there would have been every reason to expect that a few bubbles should have appeared on the electrodes, especially on the negative one, if it were only because of its action as a nucleus on the solution supposed to be formed; but none appeared even after twelve days.

973. When a few drops only of nitric acid were added to the vessel A, fig. 12., then the results were altogether different. In less than five minutes bubbles of gas appeared on the plates $\mathrm{P}^{\prime}$ and $\mathrm{P}^{\prime \prime}$ in the second vessel. 'To prove that this was the effect of the electric current (which by trial at $e$ was found at the same time to be passing, ) the connexion at $e$ was broken, the plates $\mathrm{P}^{\prime} \mathrm{P}^{\prime \prime}$ cleared from bubbles and left in the acid of the vessel $\mathrm{B}$, for fifteen minutes: during that time no bubbles appeared upon them; but on restoring the communication at $e$, a minute did not elapse before gas appeared in bubbles upon the plates. The proof, therefore, is most full and complete, that the current excited by dilute sulphuric acid with a little nitric acid in vessel $A$, has intensity enough to overcome the chemical affinity exerted between the oxygen and hydrogen of the water in the vessel $\mathrm{B}$, whilst that excitedby dilute sulphuric acid alone has not sufficient intensity.

974. On using a strong solution of caustic potassa in the vessel $A$, to excite the current, it was found by the decomposing effects at $e$, that the current passed. But it had not 
intensity enough to decompose the water in the vessel $B$; for though left for fourteen days, during the whole of which time the current was found to be passing, still not the slightest appearance of gas appeared on the plates $\mathrm{P}^{\prime} \mathrm{P}^{\prime \prime}$, nor any other signs of the water having suffered decomposition.

975. Sulphate of soda in solution was then experimented with, for the purpose of ascertaining with respect to it, whether a certain electrolytic intensity was also required for its decomposition in this state, in analogy with the result established with regard to water (974.). The apparatus was arranged as in fig. 13.; $P$ and $Z$ are the platina and zinc plates dipping into a solution of common salt; $a$ and $b$ are platina plates connected by wires of platina (except in the galvanometer $g$ ) with $\mathrm{P}$ and $\mathrm{Z} ; c$ is a connecting wire of platina, the ends of which can be made to rest either on the plates $a, b$, or on the papers moistened in solutions which are placed upon them; so that the passage of the current without decomposition, or with one or two decompositions, was under ready command, as far as arrangement was concerned. In order to change the anodes and cathodes at the places of decomposition, the form of apparatus fig. 14. was occasionally adopted. Here only one platina plate, $c$, was used; both pieces of paper on which decomposition was to be effected were placed upon it, the wires from $P$ and $Z$ resting upon these pieces of paper, or upon the plate $c$, according as the current with or without decomposition of the solutions was required.

976. On placing solution of iodide of potassium in paper at one of the decomposing localities, and solution of sulphate of soda at the other, so that the electric current should pass through both at once, the solution of iodide was slowly decomposed, yielding iodine at the anode and alkali at the cathode; but the solution of sulphate of soda exhibited no signs of decomposition, neither acid nor alkali being evolved from it. On placing the wires so that the iodide alone was subject to the action of the current (900.), it was quickly and powerfully decomposed; but on arranging them so that the sulphate of soda alone was subject to action, it still refused to yield up its elements. Finally, the apparatus was so arranged, under a wet bell-glass, that it could be left for twelve hours, the current passing during the whole time through a solution of sulphate of soda, retained in its place by only two thicknesses of bibulous litmus and turmeric paper. At the end of that time it was ascertained by the decomposition of iodide of potassium at the second place of action, that the current was passing and had passed for the twelve hours, and 


\section{Dr. Faraday's Experimental Researches in Electricity.}

yet no trace of acid or alkali from the sulphate of soda appeared.

977. From these experiments it may, I think, be concluded, that a solution of sulphate of soda can conduct a current of electricity, which is unable to decompose the neutral salt present; that this salt in the state of solution, like water, requires a certain electrolytic intensity for its decomposition; and that the necessary intensity is much higher for this substance than for the iodide of potassium in a similar state of solution.

978. I then experimented on bodies rendered decomposable by fusion, and first on chloride of lead. The current was excited by dilute sulphuric acid without any nitric acid between zinc and platina plates, fig. 15., and was then made to traverse a little chloride of lead fuseci upon glass at $a$, a paper moistened in solution of iodide of potassium at $b$, and a galvanometer at $g$. The metallic terminations at $a$ and $b$ were of platina. Being thus arranged, the decomposition at $b$ and the deflection at $g$ showed that an electric current was passing, but there was no appearance of decomposition at $a$, not even after a metallic communication at $b$ was established. The experiment was repeated several times, and I am led to conclude that in this case the current has not intensity sufficient to cause the decomposition of the chloride of lead; and further, that, like water (974.), fused chloride of lead can conduct an electric current having an intensity below that required to effect decomposition.

979. Chloride of silver was then placed at $a$, fig. 15., instead of chloride of lead. There was a very ready decomposition of the solution of iodide of potassium at $b$, and when metallic contact was made there, very considerable deflection of the galvanometer needle at $g$. Platina also appeared to be dissolved at the anode of the fused chloride at $a$, and there was every appearance of a decomposition having been effected there.

980. A further proof of decomposition was obtained in the following manner. The platina wires in the fused chloride at $a$ were brought very near together (metallic contact having been established at $b$ ), and left so; the deflection at the galvanometer indicated the passage of a current, feeble in its force, but constant. After a minute or two, however, the needle would suddenly be violently affected, and indicate a current as strong as if metallic contact had taken place at $a$. This I actually found to be the case, for the silver reduced by the action of the current crystallized in long delicate spiculæe, 
and these at last completed the metallic communication; and at the same time that they transmitted a more powerful current than the fused chloride, they proved that electro-chemical decomposition of that chloride had been going on. Hence it appears, that the current excited by dilute sulphuric acid between zinc and platina, has an intensity above that required to electrolyze the fused chloride of silver when placed between platina electrodes, although it has not intensity enough to decompose chloride of lead under the same circumstances.

981. A drop of water placed at $a$ instead of the fused chlorides, showed as in the former case (970.), that it could conduct a current unable to decompose it, for decomposition of the solution of iodide at $b$ occurred after some time. But its conducting power was much below that of the fused chloride of lead (978.).

982. Fused nitre at $a$ conducted much better than water : I was unable to decide with certainty whether it was electrolyzed, but I incline to think not, for there was no discoloration against the platina at the cathode. If sulpho-nitric acid had been used in the exciting vessel, both the nitre and the chloride of lead would have suffered decomposition like the water (906.).

983. The results thus supplied of conduction without decomposition, and the necessity of a certain electrolytic intensity for the separation of the ions of different electrolytes, are immediately connected with the experiments and results given in $\$ 10$. of the Fourth Series of these Researches (418.423. 444. 449.). But it will require a more exact knowledge of the nature of intensity, both as regards the first origin of the electric current, and also the manner in which it may be reduced or lowered by the intervention of larger or smaller portions of bad conductors, whether decomposable or not, before their relation can be minutely and fully understood.

984. In the case of water, the experiments I have as yet made, appear to show, that when the electric current is reduced in intensity below the point required for decomposition, then the degree of conduction is the same whether sulphuric acid, or any other of the many bodies which can affect its transferring power as an electrolyte, are present or not. Or, in other words, that the necessary electrolytic intensity for water is the same whether it be pure, or rendered a better conductor by the addition of these substances; and that for currents of less intensity than this, the water, whether pure or acidulated, has equal conducting power. An apparatus, fig. 12, was arranged with dilute sulphuric acid in the vessel A, 


\section{Dr. Faraday's Experimental Researches in Electricity.}

and pure distilled water in the vessel B. By the decomposition at $e$, it appeared as if water was a better conductor than dilute sulphuric acid for a current of such low intensity as to cause no decomposition. I am inclined, however, to attribute this apparent superiority of water to variations in that peculiar condition of the platina electrodes which is referred to further on in this Series (1040.), and which is assumed, as far as I can judge, to a greater degree in dilute sulphuric acid than in pure water. 'The power, therefore, of acids, alkalies, salts, and other bodies in solution, to increase conducting power, appears to hold good only in those cases where the electrolyte subject to the current suffers decomposition, and loses all influence when the current transmitted has too low an intensity to effect chemical change. It is probable that the ordinary conducting power of an electrolyte in the solid state (419.) is the same as that which it possesses in the fluid state for currents under the due electrolytic intensity.

985. Currents of electricity, produced by less than eight or ten series of voltaic elements, can be reduced to that intensity at which water can conduct them without suffering decomposition, by causing them to pass through three or four vessels in which water shall be successively interposed between platina surfaces. The principles of interference upon which this effect depends, will be described hereafter (1009. 1018.), but the effect may be useful in obtaining currents of standard intensity, and is probably applicable to batteries of any number of pairs of plates.

986. As there appears every reason to expect that all electrolytes will be found subject to the law which requires an electric current of a certain intensity for their decomposition, but that they will differ from each other in the degree of intensity required, it will be desirable hereafter to arrange them in a table, in the order of their electrolytic intensities. Investigations on this point must, however, be very much extended, and include many more bodies than have been here mentioned before such a table can be constructed. It will be especially needful in such experiments, to describe the nature of the electrodes used, or, if -possible, to select such as, like platina or plumbago in certain cases, shall have no power of assisting the separation of the ions to be evolved (913.).

987. Of the two modes in which bodies can transmit the electric forces, namely, that which is so characteristically exhibited by the metals, and that in which it is accompanied by decomposition, the first appears common to all bodies, although it occurs with almost infinite degrees of difference; 
the second is at present distinctive of the electrolytes. It is, however, just possible that it may hereafter be extended to the metals; for their power of conducting without decomposition may, perhaps justly, be ascribed to their requiring a very high electrolytic intensity for their decomposition.

$987 \frac{1}{2}$. The establishment of a certain electrolytic intensity being necessary before decomposition can be effected, is of great importance in all those considerations which arise regarding the probable effects of weak currents, such for instance as those produced by natural thermo-electricity, or natural voltaic arrangements. For to produce an effect of decomposition or of combination, a current must not only exist, but have a certain intensity before it can overcome the quiescent affinities opposed to it, otherwise it will be conducted, producing no permanent effects. On the other hand, the principles are also now evident by which an opposing action can be so weakened by the juxtaposition of bodies not having quite affinity enough to cause direct action between them (913.), that a very weak current shall be able to raise the sum of actions sufficiently high, and cause chemical changes to occur.

988. In concluding this division on the intensity necessary for electroly:ation, I cannot resist pointing out the following remarkable conclusion in relation to intensity generally. It would appear that when a voltaic current is produced, having a certain intensity, dependent upon the strength of the chemical affinities by which that current is excited (916.), it can decompose a particular electrolyte without relation to the quantity of electricity passed, the intensity deciding whether the electrolyte shall give way or not. If that conclusion be confirmed, then we may arrange circumstances so that the same quantity of electricity may pass in the same time, in at the same surface, into the same decomposing body in the same state, and yet differ in intensity, decomposing in one case and in the other not. For taking a source of too low an intensity to decompose, and ascertaining the quantity passed in a given time, it is easy to take another source having a sufficient intensity, and reducing the quantity of electricity from it by the intervention of bad conductors to the same proportion as the former current, and then all the conditions will be fulfilled to produce the result described.

[To be continued.] 